\title{
Genetic and early environmental predictors of adulthood self-reports of trauma
}

\author{
Alicia J. Peel, Kirstin L. Purves, Jessie R. Baldwin, Gerome Breen, Jonathan R. I. Coleman, \\ Jean-Baptiste Pingault, Megan Skelton, Abigail R. ter Kuile, Andrea Danese* and Thalia C. Eley*
}

\section{Background}

Retrospective self-reports of childhood trauma are associated with a greater risk of psychopathology in adulthood than prospective measures of trauma. Heritable reporter characteristics are anticipated to account for part of this association, whereby genetic predisposition to certain traits influences both the likelihood of self-reporting trauma and of developing psychopathology. However, previous research has not considered how gene-environment correlation influences these associations.

\section{Aims}

To investigate reporter characteristics associated with retrospective self-reports of childhood trauma and whether these associations are accounted for by gene-environment correlation.

\section{Method}

In 3963 unrelated individuals from the Twins Early Development Study, we tested whether polygenic scores for 21 psychiatric, cognitive, anthropometric and personality traits were associated with retrospectively self-reported childhood emotional and physical abuse. To assess the presence of gene-environment correlation, we investigated whether these associations remained after controlling for composite scores of environmental adversity across development.

\section{Results}

Retrospectively self-reported childhood trauma was associated with polygenic scores for autism spectrum disorder (ASD), body mass index (BMI), post-traumatic stress disorder (PTSD) and risky behaviours. When composite scores of environmental adversity were controlled for, only associations with the polygenic scores for ASD and PTSD remained significant.

\section{Conclusions}

Genetic predisposition to ASD and PTSD may increase liability to experiencing or interpreting events as traumatic. Associations between genetic predisposition for risky behaviour and BMI with self-reported childhood trauma may reflect gene-environment correlation. Studies of the association between retrospectively self-reported childhood trauma and later-life outcomes should consider that genetically influenced reporter characteristics may confound associations, both directly and through geneenvironment correlation.

\section{Keywords}

Trauma; childhood experience; genetics; autistic spectrum disorders; aetiology.

\section{Copyright and usage}

(c) The Author(s), 2022. Published by Cambridge University Press on behalf of the Royal College of Psychiatrists. This is an Open Access article, distributed under the terms of the creative Commons Attribution licence (https://creativecommons.org/ licenses/by/4.0/), which permits unrestricted re-use, distribution, and reproduction in any medium, provided the original work is properly cited.

\section{Background}

Evidence suggests that retrospectively self-reported trauma is associated with a greater risk of psychopathology in adulthood than prospective measures of trauma, such as court records. ${ }^{1,2}$ One possible explanation for the increased risk associated with retrospective reports is that they may be more open to differences in the interpretation of experiences as traumatic, the likelihood of recalling these experiences as traumatic and the willingness to report traumatic events. These differences in reporting may be influenced by individual reporter differences, such as personality traits, psychopathology or cognitive biases. ${ }^{2}$ In turn, these characteristics could also reflect or affect risk of psychopathology. In accordance with this, evidence suggests that retrospective self-reports are partly under genetic influence. ${ }^{3,4}$ Similarly, reporter characteristics including personality traits, psychopathology and cognitive biases have also been found to have a heritable component. ${ }^{5-7}$ As such, the heritability of reporter characteristics could, in part, explain the genetic component identified for retrospectively reported trauma. ${ }^{8,9}$ In a recent study of female nurses, polygenic scores representing individual genetic liability for attention-deficit hyperactivity disorder (ADHD), major depression, neuroticism, schizophrenia, bipolar disorder and autism spectrum disorder (ASD) were all associated with a higher likelihood of reporting childhood abuse in

* Joint last authors. mid-life. ${ }^{10}$ These findings suggest that associations between retrospectively self-reported childhood trauma and later psychopathology are partly confounded by genetic predisposition to these outcomes, which influence both the likelihood of reporting traumatic events and of developing a disorder. However, these methods do not take into account the effect of environmental risk. As well as acting through the individual directly, genetics also have an impact on a person's environment. This process, termed gene-environment correlation ( $\mathrm{rGE}$ ), occurs when genetic factors influence both an individual's liability for a trait and the environments that they are exposed to. ${ }^{11}$

There are three mechanisms of rGE: passive, active and evocative. Passive rGE describes the association between the genotype that a child inherits and the rearing environment that the parents create. Evocative rGE refers to an individual's genetically influenced behaviours instigating certain responses from others. Active rGE describes the association between an individual's genetically influenced traits and the environments that they select. Therefore, part of the association between polygenic scores for psychiatric traits and self-reports of trauma may be accounted for by genetic influences on the environment. However, when investigating these associations without considering environmental factors, the effects of rGE cannot be disentangled from the direct effects of genetics on interpretation and reporting.

\section{Aims}

In this study, we aimed to investigate whether the associations between heritable reporter characteristics and retrospective 
Table 1 Demographic characteristics of the Twins Early Development Study (TEDS) study participants $(n=3963)$, as compared with the full TEDS cohort and $\mathrm{UK}^{\mathrm{a}}$

\begin{tabular}{|c|c|c|c|c|c|c|c|}
\hline & $\begin{array}{l}\text { White } \\
\text { ethnicity }\end{array}$ & $\begin{array}{l}\text { Mothers with } \\
\text { A-levels or } \\
\text { higher }\end{array}$ & $\begin{array}{l}\text { Mother } \\
\text { employed }\end{array}$ & $\begin{array}{l}\text { Father } \\
\text { employed }\end{array}$ & Female & $\begin{array}{l}\text { Monozygotic } \\
\text { twins }\end{array}$ & Families, $n$ \\
\hline $\begin{array}{l}\text { TEDS study } \\
\quad \text { participants, \% }\end{array}$ & 99 & 44 & 48 & 89 & 62 & 38 & 3963 \\
\hline $\begin{array}{l}\text { TEDS full cohort, \% } \\
\text { Comparison of TEDS study } \\
\text { participants } \\
\text { and full cohort, } \chi^{2} \text { (P) } v\end{array}$ & $\begin{array}{c}92 \\
398.5709 \\
(<0.001) \\
0.12\end{array}$ & $\begin{array}{c}36 \\
169.0096 \\
(<0.001) 0.08\end{array}$ & $\begin{array}{c}43 \\
43.2511 \\
(<0.001) 0.04\end{array}$ & $\begin{array}{c}92 \\
44.4671 \\
(<0.001) 0.04\end{array}$ & $\begin{array}{c}50 \\
250.1258 \\
(<0.001) \\
0.10\end{array}$ & $\begin{array}{c}33 \\
37.8167 \\
(<0.001) 0.04\end{array}$ & 13722 \\
\hline UK census, \% & 93 & 32 & 49 & 89 & 51 & 34 & 8221 \\
\hline
\end{tabular}

self-reports of childhood trauma are accounted for by rGE. We investigated whether polygenic scores for cognitive, personality and psychopathological traits were associated with retrospective self-reports of childhood trauma. To assess the presence of rGE, we investigated whether these associations remain after controlling for environmental adversity across development. We hypothesised that findings of rGE would suggest increased risk of experiencing traumatic events. On the other hand, if associations are found while accounting for environmental adversity, this would suggest differences in appraisal of trauma linked to genetic factors.

\section{Method}

\section{Participant characteristics}

The Twins Early Development Study (TEDS) ${ }^{12}$ is a longitudinal study of over 15000 twin pairs born in England and Wales between 1994 and1996, identified through birth records. At 13 time points between birth and age 21, TEDS has collected data on the home environment, physical and mental health, personality and cognitive abilities. The study participants included a subset of unrelated TEDS twins who had both genetic data and data on self-reported trauma at age $21(n=3963)$.

The authors assert that all procedures contributing to this work comply with the ethical standards of the relevant national and institutional committees on human experimentation and with the Helsinki Declaration of 1975, as revised in 2008. Ethical approval for TEDS has been provided by the King's College London Ethics Committee (reference: PNM/09/10-104). Written informed consent was obtained from parents and from twins themselves from age 16 onwards prior to each wave of data collection.

The demographic characteristics of the study participants as compared with the full TEDS cohort are shown in Table 1 . The study sample had a higher proportion of female participants (62\%) and White individuals (99\%) compared with the full TEDS cohort, with Cramer's $V$ indicating these differences were of small effect $(V=0.10-0.12)$. Differences in zygosity and family socioeconomic measures were of less than small effect size $(V<0.10)$. The greater proportion of female participants in the study sample reflects the gradual decrease in male participants in recent waves of TEDS data collection. ${ }^{12,15}$ At first contact, participants' ethnicity was reported by parents, from the categories of 'Asian', 'Black', 'Mixed race', 'White' and 'Other'. We acknowledge that these categories are limited for representing participant identity. The full TEDS cohort remains fairly representative of the ethnic diversity of UK families in the mid-1990s when the twins were born. ${ }^{15}$ The high proportion of participants in the study sample who identified as White corresponds to all genotyped participants being of European ancestry.

\section{Measures}

\section{Childhood trauma}

Retrospectively self-reported childhood trauma was assessed during the TEDS-21 wave of data collection. ${ }^{15}$ Experiences of trauma were measured using eight items assessing emotional and physical abuse, derived from the Avon Longitudinal Study of Parents and Children 'Life at $22+$ ' questionnaire ${ }^{16}$ (Supplementary Table 1 available at https://doi.org/10.1192/bjp.2021.207). Participants reported how frequently they experienced these types of abuse during their childhood, on a scale of 'Never' (0) to 'Very often' (4), with total scores ranging from 0 to 32 . The average total score in the study sample was 5.2 (s.d. $=4.5$ ).

\section{Genome-wide polygenic scores}

Genome-wide polygenic scores for 21 relevant psychiatric, cognitive, anthropometric and personality traits derived from the largest genome-wide association study (GWAS) were selected (Supplementary Table 2). The majority of these polygenic scores had been previously constructed in TEDS for use in the prediction of cognitive and behavioural traits. ${ }^{17-19}$ Where TEDS had formed part of the discovery GWAS sample, polygenic scores were created from summary statistics generated without the contribution of the TEDS cohort. For example, polygenic scores for intelligence were created from summary statistics derived from a subset of the GWAS discovery sample (Supplementary Table 2). DNA samples obtained from saliva and buccal cheek swabs were genotyped on either Affymetrix GeneChip 6.0 or Illumina HumanOmniExpressExome$8 \mathrm{v} 1.2$ arrays and underwent common quality control procedures. Full genotyping and quality control processes as detailed elsewhere ${ }^{20}$ are provided in the Supplementary Methods. Polygenic scores were constructed using LDpred, ${ }^{21}$ with the proportion of genetic markers assumed to be contributing to the trait set to $1 .^{22}$

\section{Environmental measures}

Environmental factors capturing environmental adversity across development were selected, including maternal depression, ${ }^{23}$ economic disadvantage, ${ }^{24}$ family cohesion, ${ }^{25}$ parental feelings or discipline, ${ }^{26}$ negative life events ${ }^{26}$ and peer difficulties ${ }^{27}$ (Supplementary Table 3). These measures have previously been associated with retrospectively self-reported childhood trauma, and indicate the 
experience of challenging early environments that tend to co-occur with childhood trauma.

Early adversities were separated according to whether they were parent- or self-reported, as reports by parents and children typically show poor agreement, partly because they provide different perspectives on the family environment. ${ }^{28}$ Participants were included providing that they had data for more than $75 \%$ of variables, those missing more than $25 \%$ of variables at each age were excluded. As the nature of rGE effects vary across development, environmental adversities in pre-school (age 1-4), middle childhood (7-10) and early adolescence (12-16) were summed to create composite scores of environmental adversity in each developmental period. The collection of self-reported measures began from age 9, resulting in five composite scores: parent-reported environmental adversities in preschool, middle childhood and early adolescence, and self-reported environmental adversities in middle childhood and early adolescence (Supplementary Table 3 ).

\section{Data handling}

All predictors were standardised by centring and dividing by 2 s.d.s, resulting in a mean of 0 and a s.d. of 0.5 , using the rescale function from the R package 'arm' (Supplementary Table 4). ${ }^{29}$

To account for participants with missing environmental adversity composite scores, multiple imputation was conducted using the $\mathrm{R}$ package 'mice', using a prediction matrix containing the five composite scores, polygenic scores, outcomes and covariates. ${ }^{30,31}$ The average missingness of environmental variables in the sample was $3.6 \%$, the number of imputed data-sets was 25 , and the number of iterations was $20 .^{32}$ All analyses were then performed through 'mice' with the imputed data-sets.

\section{Analysis}

First, we assessed whether polygenic scores for psychiatric, cognitive, anthropometric and personality traits were associated with self-reported childhood trauma. Univariable linear regression models were run to assess the association between each polygenic score and retrospectively self-reported childhood trauma. Subsequently, a multivariable linear regression model was run, including all 21 polygenic scores.

Second, we tested the assumption that the composite scores of environmental adversity across development were associated with self-reported childhood trauma, as predicted from previous literature. Univariable linear regression models were run to assess the associations between each of the five environmental adversity composite scores and retrospectively self-reported childhood trauma. Then a multivariable linear regression model was run, including all five environmental adversity composite scores.

Finally, to assess the presence of rGE, we investigated whether the associations between polygenic scores and self-reported childhood trauma remained after controlling for environmental adversity across development. To do so, we regressed retrospectively self-reported childhood trauma on both the polygenic scores and the five composite scores of environmental adversity across development in one multivariable linear regression model.

As a planned control, we assessed the specificity of these findings to retrospectively self-reported childhood trauma, by comparing associations with self-reports of contemporaneous traumas in adulthood.

All analyses were conducted using the $\mathrm{lm}$ function within 'mice', in $\mathrm{R}$ version $4.0 .3 .^{33}$ Analyses were pre-registered on the Open Science Framework prior to receiving the data-set (https://osf.io/ vk5jf/).

\section{Covariates and multiple testing}

In all analyses, birth year, gender and genotyping batch were included as covariates. In analyses of polygenic scores, the first 10 genetic principal components were also included. Corrections for multiple testing were applied using the Benjamini-Hochberg false discovery rate (FDR) adjustment, with a threshold of 0.05 , which controls for the expected proportion of false positives among the significant results. ${ }^{34}$

\section{Results}

\section{Association between polygenic scores and retrospective self-reports of childhood trauma}

Figure 1 shows the associations between genome-wide polygenic scores for psychiatric, cognitive, anthropometric and personality traits with retrospectively self-reported childhood trauma at age 21. Univariable models were used to assess the associations between each of the polygenic scores and retrospectively selfreported childhood trauma. A multivariable model including all 21 polygenic scores was then used to assess the independent associations between the polygenic scores and retrospectively self-reported childhood trauma. Effect sizes $(\beta)$ are shown for a 2 s.d. increase in polygenic scores.

In univariable linear regression models, polygenic scores for ADHD, anxiety, ASD, body mass index (BMI), irritability, major depressive disorder, mood swings, neuroticism, post-traumatic stress disorder (PTSD), risky behaviours and schizophrenia were positively associated with retrospectively self-reported childhood trauma after FDR correction for multiple testing. A 2 s.d. increase in these polygenic scores was associated with an increase in selfreported childhood trauma ranging from $\beta=0.39$ (95\% CI 0.090.69 ) for anxiety to $\beta=0.79$ (95\% CI 0.46-1.11) for PTSD (Supplementary Table 5). Polygenic scores for household income ( $\beta=-0.44,95 \% \mathrm{CI}-0.74$ to -0.13$)$ and subjective well-being ( $\beta=$ $-0.48,95 \% \mathrm{CI}-0.79$ to -0.18 ) were negatively associated with self-reported childhood trauma. When all polygenic scores were included in a multivariable linear regression model, only scores for ASD ( $\beta=0.47,95 \%$ CI 0.15-0.80), BMI ( $\beta 0.41-95 \%$ CI 0.09, 0.73), PTSD ( $\beta=0.56,95 \%$ CI $0.23-0.89)$ and risky behaviours $(\beta=0.45$, $95 \%$ CI $0.14-0.76)$ remained independently associated with retrospectively self-reported childhood trauma.

\section{Association between environmental adversity across development and retrospective self-reports of childhood trauma}

Figure 2 shows the associations between the five composite scores of parent- and self-reported environmental adversity in pre-school, middle childhood and early adolescence with retrospectively selfreported childhood trauma at age 21. Univariable models were used to assess the associations between each of the environmental composite scores and retrospectively self-reported childhood trauma. A multivariable model including all five environmental composite scores was then used to assess the independent associations between the composite scores and retrospectively self-reported childhood trauma. Effect sizes $(\beta)$ are shown for a 2 s.d. increase in composite scores.

As anticipated, in univariable linear regression models all five composite scores of environmental adversity were associated with retrospectively self-reported childhood trauma. A 2 s.d. increase in any composite score was associated with an increase in selfreported childhood trauma ranging from $\beta=1.47$ (95\% CI 1.131.81) for parent-reported environmental adversity in pre-school 


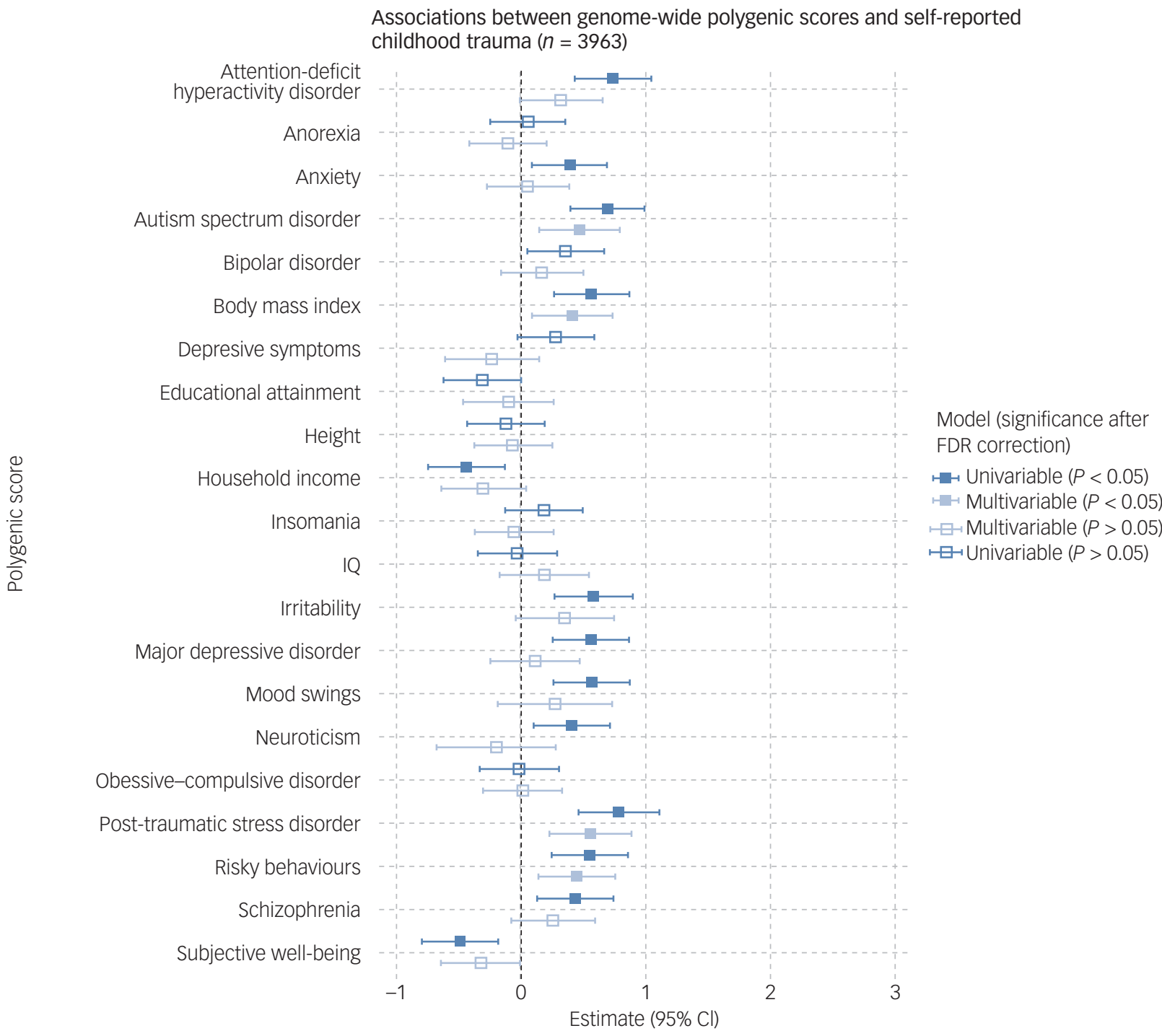

Fig. 1 Associations between genome-wide polygenic scores and retrospectively self-reported childhood trauma at age 21, derived from univariable and multivariable linear regression models $(n=3963)$.

Associations between environmental adversity across development and self-reported childhood trauma $(n=3963)$

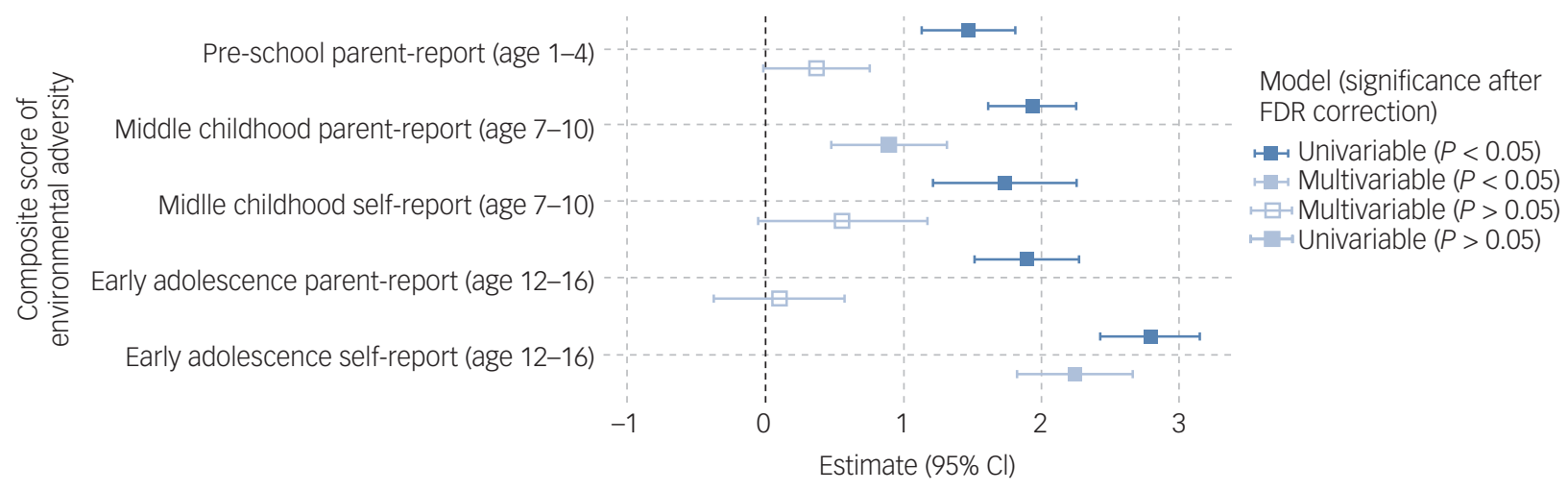

Fig. 2 Associations between composite scores of parent- and self-reported environmental adversity across development and retrospectively self-reported childhood trauma at age 21, derived from univariable and multivariable linear regression models $(n=3963)$. 
Associations between environmental adversity across development and genome-wide polygenic scores with self-reported childhood trauma $(n=3963)$

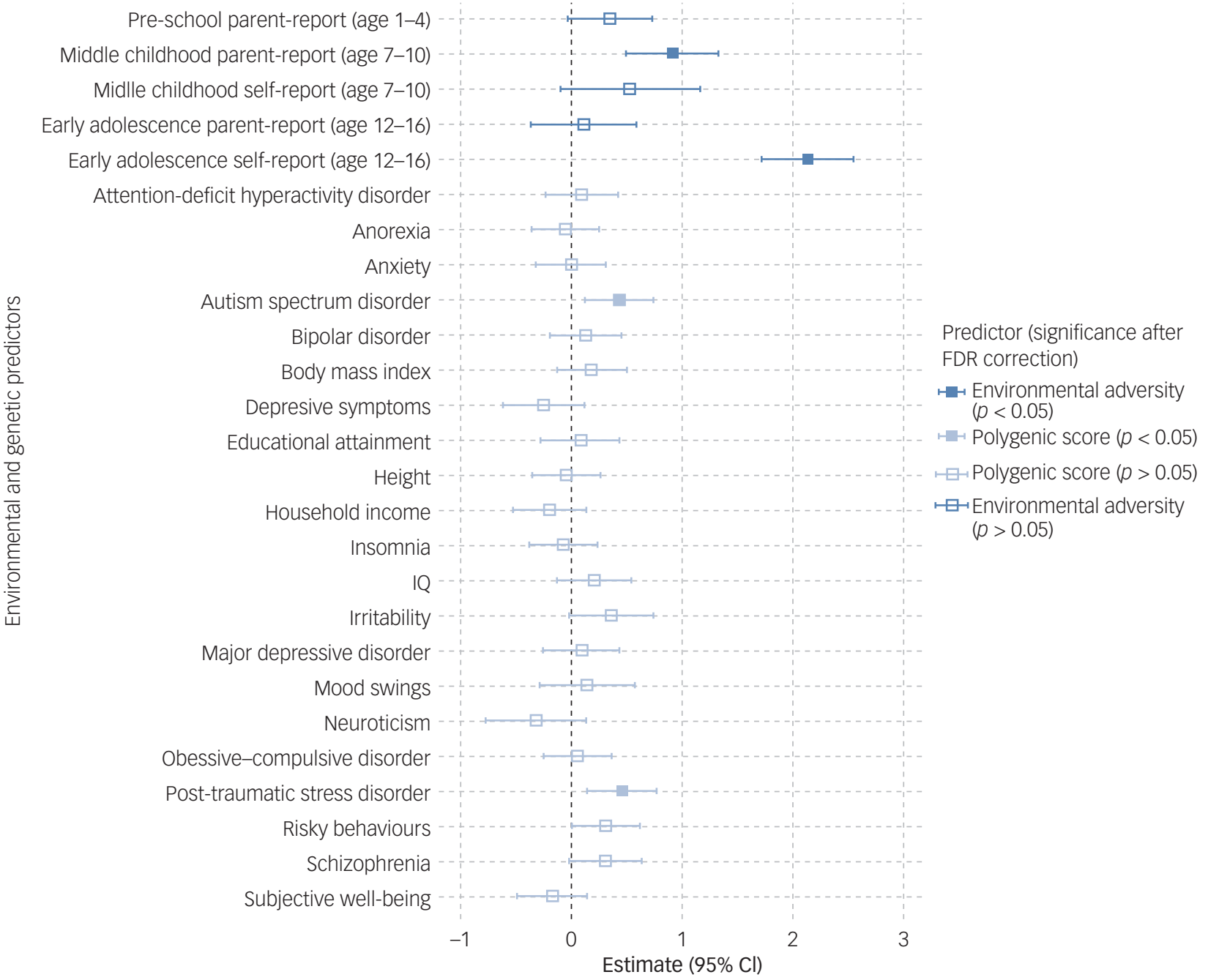

Fig. 3 Associations between composite scores of parent- and self-reported environmental adversity across development and genome-wide polygenic scores with retrospectively self-reported childhood trauma at age 21, derived from a multivariable linear regression model $(n=3963)$.

to $\beta=2.78$ (95\% CI $2.42-3.15$ ) for self-reported environmental adversity in early adolescence.

When all composite scores were included in a multivariable linear regression model, only two remained independently associated with retrospectively self-reported childhood trauma. Self-reported environmental adversity in early adolescence, which captured home chaos, parental feelings and discipline, parental monitoring and control, life events and peer victimisation (Supplementary Table 3), was most strongly associated with self-report childhood trauma ( $\beta=2.24,95 \%$ CI 1.83-2.66). Parent-reported environmental adversity in middle childhood also remained associated, which captured changes in parental marital status or household finances, parental feelings and discipline, life events and child relationship problems $(\beta=0.90,95 \%$ CI $0.48-1.32)$.

\section{Independent associations between polygenic scores and environmental adversity across development with retrospective self-reports of childhood trauma}

To assess the presence of rGE, we investigated whether the associations between polygenic scores and self-reported childhood trauma remained after controlling for environmental adversity across development (Fig. 3). We regressed retrospectively self-reported childhood trauma on the 21 polygenic scores and the five composite scores of environmental adversity across development in one multivariable model. Effect sizes $(\beta)$ are shown for a 2 s.d. increase in composite scores and polygenic scores.

In the model of environmental composite scores and polygenic scores, only parent-reported environmental adversity in middle childhood ( $\beta=0.91,95 \%$ CI 0.49-1.33), self-reported environmental adversity in early adolescence $(\beta=2.13,95 \%$ CI $1.72-2.55)$ and the polygenic scores for ASD $(\beta=0.43,95 \% \mathrm{CI} 0.13-0.74)$ and PTSD ( $\beta=0.46,95 \%$ CI $0.15-0.77)$ remained independently associated with retrospectively self-reported childhood trauma.

Planned control analyses assessing the specificity of the findings to retrospectively self-reported childhood trauma indicated a different pattern of associations for self-reports of contemporaneous traumas in adulthood (Supplementary Table 6). Self-reported environmental adversity in early adolescence was associated with both self-reported partner abuse $(\beta=2.00,95 \%$ CI 1.45-2.54) and major life events $(\beta=1.26,95 \%$ CI $0.90-1.62)$. Parent-reported environmental adversity in pre-school $(\beta=0.41,95 \%$ CI 0.10 0.72 ) was additionally associated with self-reported major life events. For both self-reports of contemporaneous adulthood 
traumas, the polygenic scores for educational attainment and major depressive disorder were the most strongly associated, however, no polygenic scores remained significantly associated with either of the self-reported adulthood traumas after FDR correction for multiple testing.

\section{Discussion}

\section{Main findings}

In this study we examined polygenic scores associated with retrospective self-reports of childhood trauma and whether these associations remain after controlling for environmental adversity across development. Retrospectively self-reported childhood trauma was independently associated with polygenic scores for ASD, BMI, PTSD and risky behaviours. When environmental adversity was controlled for, only the polygenic scores for ASD and PTSD remained significantly associated.

\section{Interpretation and comparison with findings from other studies}

The robust association between retrospectively self-reported childhood trauma and polygenic scores for ASD replicates previous findings from two cohorts. ${ }^{10,35}$ There are two possible explanations for this finding. One explanation is $\mathrm{rGE}$, when genetic factors influence both an individual's liability for ASD and their environments. ${ }^{11}$ Genetic liability for autistic traits may increase difficulty with social interaction, which could evoke adverse reactions, such as neglect or abuse, from others (evocative rGE), or increase the likelihood of being in dangerous environments (active rGE). ${ }^{35}$

Consistent with this explanation, another study found over transmission of genetic risk for reporting childhood trauma from parents to children with autism but not to their siblings who did not have autism. ${ }^{4}$ This suggests that the increased genetic risk of reporting childhood maltreatment in individuals with ASD may be partly explained by increased active and evocative rGE. A second explanation is that individuals with high genetic risk for ASD may be more sensitive to experiencing, interpreting and/or reporting an event as traumatic. ${ }^{35}$ This explanation is more in line with the results of the present study, as the association between the polygenic score for ASD and retrospectively self-reported childhood trauma remained significant after controlling for environmental adversity in childhood and adolescence. Consistent with this explanation, the polygenic score for ASD was not found to be associated with prospectively measured childhood trauma. ${ }^{36}$ Indeed, adults with ASD report a broad range of life events as being traumatic. ${ }^{37}$ Furthermore, they are at increased risk of developing a trauma-related disorder, even following events that would not meet the DSM ${ }^{38}$ criteria for trauma. ${ }^{37}$ This explanation does not imply that these events were not traumatic, as trauma is not only the nature of an event but also how it is experienced and recalled by the individual. Rather, it provides a framework for understanding how the subjective experience relates to the outcomes of reported trauma.

The finding that the polygenic score for PTSD remained associated with self-report childhood trauma after controlling for environmental adversity also indicates that genetic risk for PTSD may influence the subjective experience or reporting of traumatic events. This is in line with previous research showing that the PTSD polygenic score is not associated with trauma exposure severity. ${ }^{39}$ This provides evidence against rGE, whereby genetic risk for PTSD would be expected to contribute to greater exposure to traumatic events. ${ }^{39}$ These findings instead imply that individuals with genetic risk for PTSD may be more likely to interpret experiences as being traumatic. Indeed, PTSD is associated with increased processing of negative information compared with neutral stimuli, including greater attention towards and difficulty disengaging from negative stimuli, greater likelihood of interpreting neutral stimuli as threatening, and a bias towards remembering traumarelevant information. ${ }^{40}$ These information processing biases may increase the likelihood of reporting events as being traumatic, a necessary criterion for a PTSD diagnosis. ${ }^{38}$

Our finding that polygenic scores for BMI and risky behaviours were no longer significant once controlling for environmental adversity likely reflects overlap in some of the mechanisms that these variables capture. The polygenic score for risky behaviours was derived from the first principal component of behaviours across the four domains of automobile speeding propensity, number of alcoholic drinks consumed per week, whether one has ever been a smoker, and number of lifetime sexual partners, capturing a general tendency towards risk taking. ${ }^{41}$ Risk-taking behaviours and high BMI are anticipated to share behavioural mechanisms, owing to common associations with impulsivity, self-control and reward-seeking behaviours. ${ }^{42}$ These results suggest that rGE may account for some of the association between polygenic scores for these traits and retrospectively self-reported childhood trauma. This could be through passive rGE, for example, children in households where parents engage in more risky behaviours, such as alcohol misuse, are both more likely to experience childhood adversity ${ }^{43}$ and inherit a predisposition to these behaviours. These associations may also be influenced by evocative rGE, for example, impulsive behaviour in children is associated with negative parenting and harsh discipline strategies. ${ }^{44}$ In line with these mechanisms, our findings suggest that some of the effects of genotype on selfreporting of trauma are no longer present once the effects of genotype on the environment are controlled for.

Associations between retrospectively self-reported childhood trauma and genetic risk for ASD, BMI, PTSD and risky behaviours reflect the previously identified shared genetic architecture of these traits. ${ }^{9,42}$ However, unlike previous research, we did not find associations between retrospectively self-reported childhood trauma and polygenic risk for other psychopathological and personality traits. In a cohort of female nurses, in addition to the polygenic score for ASD, associations were found between self-reported childhood trauma and polygenic scores for major depressive disorder, bipolar disorder, neuroticism, schizophrenia and ADHD. ${ }^{10} \mathrm{~A}$ likely reason for this inconsistency is that the larger sample size of the nurse cohort $(n=11315)$ resulted in higher power to detect small effects (OR range 1.05-1.19). Nevertheless, in the study of nurses, when polygenic scores were created with only variants uniquely associated with each trait, the score most strongly associated with reported childhood trauma was that of ASD. ${ }^{10}$ Therefore, both studies indicate a robust association between genetic liability for ASD and retrospectively self-reported childhood trauma, independent of genetic liability for other traits, as assessed through polygenic scores. Planned control analyses indicate that this effect is specific to retrospectively reported childhood trauma, as the same associations were not observed for self-reports of contemporaneous traumas in adulthood.

\section{Limitations}

One limitation of this study was the use of composite scores of environmental adversity, meaning it is difficult to infer which specific adversities within each developmental period are driving associations with self-reported trauma. Furthermore, not all types of environmental adversity were assessed at each time point. Prenatal complications and maternal depression were only assessed in pre-school by parent-report, relationship problems were only 
assessed in middle childhood by parent-report, and peer victimisation was only assessed in early adolescence. Therefore, associations specific to a particular developmental stage could be confounded by the types of adversity assessed at that time. However, this approach enabled the inclusion of a broader scope of environmental adversities across development. Another limitation was the conceptualisation of childhood trauma as physical or emotional abuse. Childhood trauma can take several other forms, including physical and emotional neglect and sexual abuse, which are associated with differential reporting patterns, heritability estimates and outcomes. ${ }^{1,3,10}$ Nevertheless, different types of childhood trauma are found to have substantial genetic overlap. ${ }^{4}$

Finally, female participants and participants self-identifying as White were over-represented in the study sample, which may have an impact on the applicability of these findings to the general population. In the case of gender representativeness, this reflects greater retention of female participants compared with male ones in TEDS from the early teenage years onwards. To help mitigate this selection bias, gender was included as a covariate in all analyses. Ethnic diversity is a more pressing issue as only participants of European ancestry are currently included in the genotyped subset of TEDS participants, resulting in $99 \%$ of the study participants identifying as White. This is because many polygenic scores having poor predictive performance in individuals from more diverse ancestry groups, as the GWAS that these scores are derived from only include individuals of European ancestry in order to increase homogeneity. The need for large-scale GWAS in diverse human populations, and polygenic scores with predictive power across ancestral groups, is an immediate priority for research in this field. ${ }^{45}$

\section{Implications}

These findings have clear implications for understanding of retrospectively self-reported childhood trauma. They indicate that self-reports of trauma are associated with heritable reporter characteristics. This reinforces the importance of using genetically sensitive designs when assessing associations between self-reported experiences and later-life outcomes. Research that does not account for the confounding role of genetics should take particular care not to draw causal inferences. In contrast, genetically sensitive designs can help disentangle the complex mechanisms through which selfreports of childhood trauma are linked with poor outcomes, informing preventative and therapeutic strategies. Clinically, these findings suggest that an increased genetic liability for ASD and PTSD might bias the perception of life events, making individuals more sensitive to experiencing, interpreting and/or reporting an event as traumatic. Therefore, a broad range of experiences may be considered traumatic for these individuals, who may need clinical support even if diagnostic criteria for trauma are not met. ${ }^{38}$ It will be important to identify the specific cognitive biases linked to genetic liability for ASD and PTSD. A better understanding of the subjective experience of trauma will help identify possible vulnerability factors for psychopathology and targets for novel treatments. ${ }^{1}$ The use of polygenic scores alongside other established factors could help guide treatment choices based on such cognitive biases. Finally, it is important to stress that these results show only associations with self-reported experiences and do not imply that genetic predisposition for these traits would cause a person to experience adversity.

Alicia J. Peel (D), Social, Genetic and Developmental Psychiatry Centre; Institute of Psychiatry, Psychology \& Neuroscience, King's College London, UK; Kirstin L. Purves (D), Social, Genetic and Developmental Psychiatry Centre; Institute of Psychiatry, Psychology \& Neuroscience, King's College London, UK; Jessie R. Baldwin (D), Social, Genetic and Developmental Psychiatry Centre; Institute of Psychiatry, Psychology \& Neuroscience, King's College London, UK; and Division of Psychology and Language Sciences, Department of Clinical, Educational and Health
Psychology, University College London, UK; Gerome Breen (D), Social, Genetic and Developmental Psychiatry Centre; Institute of Psychiatry, Psychology \& Neuroscience King's College London, UK; and UK National Institute for Health Research (NIHR) Biomedical Research Centre, South London and Maudsley NHS Trust, UK; Jonathan R. I. Coleman (D), Social, Genetic and Developmental Psychiatry Centre; Institute of Psychiatry, Psychology \& Neuroscience, King's College London, UK; and UK National Institute for Health Research (NIHR) Biomedical Research Centre, South London and Maudsley NHS Trust, UK; Jean-Baptiste Pingault (D), Social, Genetic and

Developmental Psychiatry Centre; Institute of Psychiatry, Psychology \& Neuroscience, King's College London, UK; and Division of Psychology and Language Sciences, Department of Clinical, Educational and Health Psychology, University College London, UK; Megan Skelton (D), Social, Genetic and Developmental Psychiatry Centre; Institute of Psychiatry. Psychology \& Neuroscience, King's College London, UK and UK National Institute for Health Research (NIHR) Biomedical Research Centre, South London and Maudsley NHS Trust, UK; Abigail R. ter Kuile (D), Social, Genetic and Developmental Psychiatry Centre; Institute of Psychiatry, Psychology \& Neuroscience, King's College London, UK; and UK National Institute for Health Research (NIHR) Biomedical Research Centre, South London and Maudsley NHS Trust, UK; Andrea Danese (D), Social, Genetic and Developmental Psychiatry Centre; Institute of Psychiatry, Psychology \&

Neuroscience, King's College London, UK; Department of Child \& Adolescent Psychiatry, Institute of Psychiatry, Psychology \& Neuroscience, King's College London, UK; and

National and Specialist CAMHS Trauma, Anxiety, and Depression Clinic, South London and Maudsley NHS Foundation Trust, UK; Thalia C. Eley (D), Social, Genetic and Developmental Psychiatry Centre; Institute of Psychiatry, Psychology \& Neuroscience, King's College London, UK; and UK National Institute for Health Research (NIHR) Biomedical Research Centre, South London and Maudsley NHS Trust, UK

Correspondence: Thalia C. Eley. Email: thalia.eley@kcl.ac.uk

First received 6 Jul 2021, final revision 16 Nov 2021, accepted 2 Dec 2021

\section{Supplementary material}

To view supplementary material for this article, please visit https://doi.org/10.1192/bjp.2021.207.

\section{Data availability}

The data that support the findings of this study came from the Twins Early Development Study (TEDS). Eligible researchers can apply for access to the TEDS data: https://www.teds.ac.uk/ researchers/teds-data-access-policy.

\section{Acknowledgements}

We gratefully acknowledge the ongoing contribution of the participants in the Twins Early Development Study (TEDS) and their families.

\section{Author contributions}

All authors were involved in formulating the research questions and devising the analysis plan Analysis was led by A.JP. with support from K.L.P. J R.IC. and M.S. All authors contributed to interpretation of results. A.J.P. wrote the first draft of the manuscript. All authors provided critical feedback on manuscript drafts and approved the final version. T.C.E. and A.D. jointly supervised this work.

\section{Funding}

TEDS is supported by a programme grant to T.C.E. and G.B. from the UK Medical Research Council (MR/V012878/1 and previously MR/M021475/1). A.D. is part funded by the National Institute for Health Research (NIHR) Biomedical Research Centre at South London and Maudsley NHS Foundation Trust and King's College London. A.JP is supported by an ESRC studentship. M.S. Fond A.R.TK are funded by NIHR Maudsley Biomedical Research Centre studentships. J.-B.P. is supported by the Medical Research Foundation 2018Em orging Leaders 1 st Prize in Adolescent Mental Health (MRF-160-0002-ELP-PINGA). J.R.B. is funded by a Wellcome Trust Sir Henry Wellcome fellowship (grant 215917/Z/19/Z). This study represents independent research part funded by the NIHR Maudsley Biomedical Research Centre at South London and Maudsley NHS Foundation Trust and King's College London. The views expressed are those of the author(s) and not necessarily those of the NHS, the NIHR or the Department of Health and Social Care.

\section{Declaration of interest}

The authors declare no conflict of interest. 


\section{References}

1 Danese A, Widom CS. Objective and subjective experiences of child maltreatment and their relationships with psychopathology. Nat Hum Behav 2020; 4: 811-18

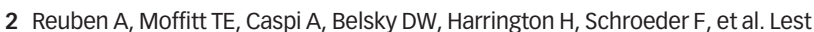
we forget: comparing retrospective and prospective assessments of adverse childhood experiences in the prediction of adult health. J Child Psychol Psychiatry 2016; 57: 1103-12.

3 Schulz-Heik RJ, Rhee SH, Silvern L, Lessem JM, Haberstick BC, Hopfer C, et al. Investigation of genetically mediated child effects on maltreatment Behav Genet 2009; 39: 265-76.

4 Warrier V, Kwong ASF, Luo M, Dalvie S, Croft J, Sallis HM, et al. Gene-environment correlations and causal effects of childhood maltreatment on physical and mental health: a genetically informed approach. Lancet Psychiatry $2021 ; 8$ : 373-86.

5 Vukasović T, Bratko D. Heritability of personality: a meta-analysis of behavior genetic studies. Psychol Bull 2015; 141: 769-85.

6 Pettersson $\mathrm{E}$, Larsson $\mathrm{H}$, Lichtenstein $\mathrm{P}$. Common psychiatric disorders share the same genetic origin: a multivariate sibling study of the Swedish population. Mol Psychiatry 2016; 21: 717-21.

7 Zavos HMS, Rijsdijk FV, Gregory AM, Eley TC. Genetic influences on the cognitive biases associated with anxiety and depression symptoms in adolescents. J Affect Disord 2010; 124: 45-53.

8 Coleman JRI, Peyrot WJ, Purves KL, Davis KAS, Rayner C, Choi SW, et al. Genome-wide gene-environment analyses of major depressive disorder and reported lifetime traumatic experiences in UK Biobank. Mol Psychiatry 2020 25: $1430-46$

9 Dalvie S, Maihofer AX, Coleman JRI, Bradley B, Breen G, Brick LA, et al. Genomic influences on self-reported childhood maltreatment. Trans/ Psychiatry 2020; 10: 38.

10 Ratanatharathorn A, Koenen KC, Chibnik LB, Weisskopf MG, Rich-Edwards JW Roberts AL. Polygenic risk for autism, attention-deficit hyperactivity disorder schizophrenia, major depressive disorder, and neuroticism is associated with the experience of childhood abuse. Mol Psychiatry 2021; 26: 1696-705

11 Plomin R, DeFries JC, Loehlin JC. Genotype-environment interaction and correlation in the analysis of human behavior. Psychol Bull 1977; 84: 309-22.

12 Rimfeld K, Malanchini M, Spargo T, spickernell G, Selzam S, McMillan A, et al. Twins early development study: a genetically sensitive investigation into behavioral and cognitive development from infancy to emerging adulthood. Twin Res Hum Genet 2019; 22: 508-13.

13 Office of National Statistics. Living in Britain: Results from the 2000 General Household Survey. The Stationery Office, 2001.

14 Imaizumi Y. A comparative study of zygotic twinning and triplet rates in eight countries, 1972-1999. J Biosoc Sci 2003; 35: 287-302.

15 Rimfeld K, Malanchini M, Packer AE, Gidziela A, Allegrini AG, Ayorech Z, et al. The winding roads to adulthood: a twin study. JCPP Adv 2021; 1(4): e12053.

16 Houtepen LC, Heron J, Suderman MJ, Tilling K, Howe LD. Adverse childhood experiences in the children of the avon longitudinal study of parents and children (ALSPAC). Wellcome Open Res 2018; 3: 106.

17 Gidziela A, Rimfeld K, Malanchini M, Allegrini AG, McMillan A, Selzam S, et al. Using DNA to predict behaviour problems from preschool to adulthood. J Child Psychol Psychiatr [Epub ahead of print] 6 Sept 2021. Available from: https://doi. org/10.1111/jcpp.13519

18 Allegrini AG, Karhunen V, Coleman JRI, Selzam S, Rimfeld K, von Stumm S, et al. Multivariable G-E interplay in the prediction of educational achievement. PLOS Genet 2020; 16: e1009153.

19 Allegrini A, Selzam S, Rimfeld K, von Stumm S, Pingault J-B, Plomin R. Genomic prediction of cognitive traits in childhood and adolescence. Mol psychiatry 2019; 24: 819-27.

20 Selzam S, McAdams TA, Coleman JRI, Carnell S, O'Reilly PF, Plomin R, et al. Evidence for gene-environment correlation in child feeding: links between common genetic variation for BMI in children and parental feeding practices. PLOS Genet 2018; 14: e1007757.

21 Vilhjálmsson BJ, Yang J, Finucane HK, Gusev A, Lindström S, Ripke S, et al. Modeling linkage disequilibrium increases accuracy of polygenic risk scores. Am J Hum Genet 2015; 97: 576-92.

22 Selzam S, Ritchie SJ, Pingault J-B, Reynolds CA, O'Reilly PF, Plomin R. Comparing within- and between-family polygenic score prediction. Am J Hum Genet 2019; 105: 351-63.
23 Doidge JC, Higgins DJ, Delfabbro P, Segal L. Risk factors for child maltreatment in an Australian population-based birth cohort. Child Abuse Neg/ 2017; 64: 47-60.

24 Doidge JC, Higgins DJ, Delfabbro P, Edwards B, Vassallo S, Toumbourou JW et al. Economic predictors of child maltreatment in an Australian populationbased birth cohort. Child Youth Serv Rev 2017; 72: 14-25.

25 Stith SM, Liu T, Christopher Davies L, Boykin EL, Alder MC, Harris JM, et al. Risk factors in child maltreatment: a meta-analytic review of the literature. Aggress Violent Behav 2009; 14: 13-29.

26 Brown J, Cohen P, Johnson JG, Salzinger S. A longitudinal analysis of risk factors for child maltreatment: findings of a 17-year prospective study of officially recorded and self-reported child abuse and neglect. Child Abuse Neg/ 1998; 22: $1065-78$

27 Radford L, Corral S, Bradley C, Fisher HL. The prevalence and impact of child maltreatment and other types of victimization in the UK: findings from a population survey of caregivers, children and young people and young adults. Child Abuse Neg/ 2013; 37: 801-13.

28 Sessa FM, Avenevoli S, Steinberg L, Morris AS. Correspondence among informants on parenting: preschool children, mothers, and observers. J Fam Psychol 2001; 15: 53-68.

29 Gelman A. Scaling regression inputs by dividing by two standard deviations Stat Med 2008; 27: 2865-73.

30 Moons KGM, Donders RART, Stijnen T, Harrell Jr FE. Using the outcome for imputation of missing predictor values was preferred. J Clin Epidemiol 2006; 59: 1092-101.

31 Zhang Z. Multiple imputation with multivariate imputation by chained equation (MICE) package. Ann Transl Med 2016; 4(2): 30.

32 van Buuren S. Flexible Imputation of Missing Data, Second Edition. CRC Press, 2018

33 R Core Team. R: A Language and Environment for Statistical Computing. R Foundation for Statistical Computing, 2021 (https://www.R-project.org/).

34 Benjamini $Y$, Hochberg $Y$. Controlling the false discovery rate: a practical and powerful approach to multiple testing. J R Stat Soc Series B Stat Methodol 1995; 57: 289-300.

35 Warrier V, Baron-Cohen S. Childhood trauma, life-time self-harm, and suicida behaviour and ideation are associated with polygenic scores for autism. Mol Psychiatry 2021; 26: 1670-84.

36 Sallis HM, Croft J, Havdahl A, Jones HJ, Dunn EC, Davey Smith G, et al. Genetic liability to schizophrenia is associated with exposure to traumatic events in childhood. Psychol Med 2020; 1: 1-8.

37 Rumball F, Happé F, Grey N. Experience of trauma and PTSD symptoms in autistic adults: risk of PTSD development following DSM-5 and non-DSM-5 traumatic life events. Autism Res 2020; 13: 2122-32.

38 American Psychiatric Association. Diagnostic and Statistical Manual of Mental Disorders (DSM-5®). American Psychiatric Pub, 2013.

39 Waszczuk MA, Docherty AR, Shabalin AA, Miao J, Yang X, Kuan PF, et al. Polygenic prediction of PTSD trajectories in 9/11 responders. Psychol Med [Epub ahead of print] 23 Oct 2020. Available from: https://doi.org/10.1017/ S0033291720003839

40 Bomyea J, Johnson A, Lang AJ. Information processing in PTSD: evidence for biased attentional, interpretation, and memory processes. Psychopathol Rev 2017; 4: 218-43.

41 Linnér R K, Biroli $\mathrm{P}$, Kong $\mathrm{E}$, Meddens SFW, Wedow R, Fontana MA, et al Genome-wide association analyses of risk tolerance and risky behaviors in over 1 million individuals identify hundreds of loci and shared genetic influences. Nat Genet 2019; 51: 245-57.

42 Clifton EAD, Perry JRB, Imamura F, Lotta LA, Brage S, Forouhi NG, et al. Genome-wide association study for risk taking propensity indicates shared pathways with body mass index. Commun Biol 2018; 1: 36 .

43 Dube SR, Anda RF, Felitti VJ, Croft JB, Edwards VJ, Giles WH. Growing up with parental alcohol abuse: exposure to childhood abuse, neglect, and household dysfunction. Child Abuse Negl 2001; 25: 1627-40.

44 Gershoff ET. Corporal punishment by parents and associated child behaviors and experiences: a meta-analytic and theoretical review. Psychol Bull 2002 128: $539-79$.

45 Duncan L, Shen H, Gelaye B, Meijsen J, Ressler K, Feldman M, et al. Analysis of polygenic risk score usage and performance in diverse human populations. Nat Commun 2019: 10: 1-9. 